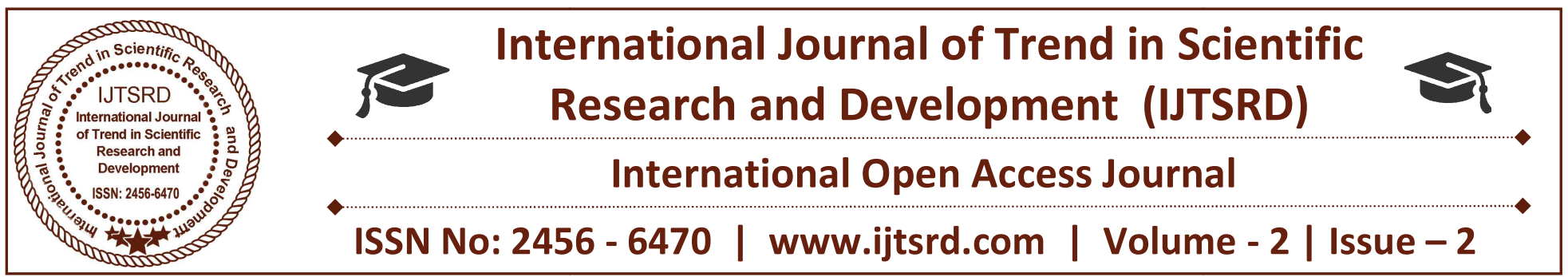

\title{
Smart Dust Cubic-Millimetre Computer for Shrewd Farm
}

\author{
Dr. I. Lakshmi \\ Assistant Professor, Department of Computer science, \\ Stella Maris College, Chennai, India
}

\begin{abstract}
One of the Major issues which we confront today is agribusiness. In the event that the agribusiness is done in a substantial scale the checking of vast ranches turns into a dreary procedure, so a computational strategy which screens the supplement substance of the distinctive parts of the homestead land is required to improve the harvest yield of the homestead arrive. so my point is to momentarily screen the homestead arrive constantly utilizing another innovation called keen clean and send the data for handling and if there is an absence of a specific supplement then consequently the required measure of the supplement is blended with the water and afterward inundated, and if the ranch needs in water then it is detected by the brilliant tidy and data is transmitted, then the pump is naturally exchanged on. By utilizing this innovation the homestead can be checked 360 degrees which will then outcome in expanded product yield.
\end{abstract}

Index: smart dust requirements sensors and motors, computing at the millimetre scale, low energy consumption, remote programmability, communicating from a grain of sand, optical communications, passive reflective systems- active steered laser systems, listening to a dust field, advantages of imaging receivers

\section{INTRODUCTION}

The Smart Dust project is probing micro fabrication technology's limitations to determine whether an autonomous sensing, computing, and communication system can be packed into a cubic-millimetre mote to form the basis of integrated, massively distributed sensor networks. Decreasing computing device size,

increased connectivity, and enhanced interaction with the physical world have characterized computing history. Recently, the popularity of small computing devices, such as handheld computers and cell phones, burgeoning Internet growth, and the diminishing size and cost of sensors - especially transistors - have accelerated these trends. The emergence of small computing elements, with sporadic connectivity and increased interaction with the environment, provides enriched opportunities to reshape interactions between people and computers and spur ubiquitous computing research.1 The Smart Dust project2 is exploring whether an autonomous sensing, computing, and communication system can be packed into a cubicmillimetre mote (a small particle or speck) to form the basis of integrated, massively distributed sensor networks. Although we've chosen a somewhat arbitrary size for our sensor systems, exploring micro fabrication technology's limitations is our fundamental goal. Because of its discrete size, substantial functionality, connectivity, and anticipated low cost, Smart Dust will facilitate innovative methods of interacting with the environment, providing more information from more places less intrusively. We use Smart Dust to pursue projects such as

$>$ deploying defence networks rapidly by unmanned aerial vehicles or artillery;

monitoring rotating-compression-blade high cycle fatigue;

tracking the movements of birds, small animals, and insects;

monitoring environmental conditions that affect crops and livestock; 
$>$ building virtual keyboards;

$>$ managing inventory control;

$>$ monitoring product quality;

$>$ constructing smart-office spaces; and

$>$ providing interfaces for the disabled.

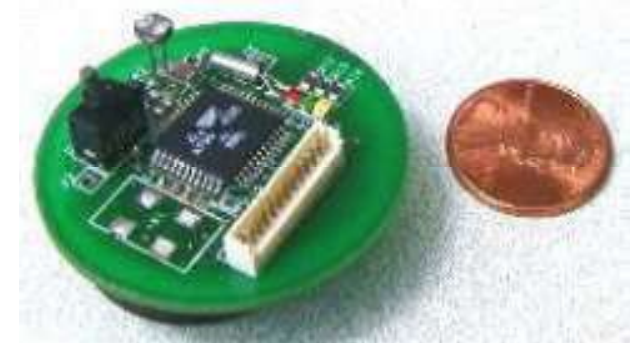

\section{SMART DUST REQUIREMENTS}

Brilliant Dust requires both developmental and progressive advances in scaling down, coordination, and vitality administration. Architects can utilize miniaturized scale electromechanical frameworks (MEMS) to assemble little sensors, optical correspondence parts, and power supplies, while microelectronics gives expanding usefulness in littler zones, with lower vitality utilization. Figure 1 demonstrates the applied outline of a Smart Dust bit. The power framework comprises of a thick-film battery, a sun oriented cell with a chargeincorporating capacitor for times of dimness, or both. Contingent upon its target, the outline coordinates different sensors, including light, temperature, vibration, attractive field, acoustic, and wind shear, onto the bit. A coordinated circuit gives sensor-flag preparing, correspondence, control, information stockpiling, and vitality administration. A photodiode permits optical information gathering. We are directly investigating two transmission plans: uninvolved transmission utilizing a corner-3D square retro reflector, and dynamic transmission utilizing a laser diode and steerable mirrors. The bit's tiny size makes vitality administration a key part. Current battery and capacitor innovation stores roughly 1 joule for every cubic $\mathrm{mm}$ and 10 mill joules per cubic $\mathrm{mm}$, separately, though sun powered cells give 1 joule for every day per square $\mathrm{mm}$ in daylight and 1 to 10 mill joules every day for every square $\mathrm{mm}$ inside. Our optical collector devours roughly 0.1 nanojoule per bit, and the transmitter utilizes 1 nanojoule per bit. We anticipate that our simple will computerized converter to require 1 nanojoule per test and calculations to devour under 1 picojoule per guideline, rather than present processors, for example, the CoolRisc 813 centre, which utilizes 22 picojoules per direction, and the StrongARM SA1100, which expends around 1 nanojoule per direction. These assessments show that for each sensor test or transmission, we can perform around 1,000 8-bit operations, so it is favourable to trade additional counts for less examples or transmitted bits. Further, given our 1 mill joule every day of vitality from indoor lighting, each second we can test a sensor, consider the outcome, and transmit a few information. To decide our exploration gauge and rapidly create equipment for testing organizing calculations, we utilized business off-the-rack equipment to manufacture a progression of remote sensor hubs. We utilized either optical or radio-recurrence correspondence models to create one-cubic-inch gadgets. Besides, other research bunches have utilized these bits to build up a minor working framework and send a 100-hub organize.

\section{AI SENSORS AND MOTORS}

The multibillion-dollar MEMS industry has been developing for a very long while, with real markets in car weight sensors and accelerometers, medicinal sensors, and process control sensors. Late advances in innovation have put huge numbers of these sensor forms on exponentially diminishing size/control/cost bends. What's more, varieties of MEMS sensor innovation are utilized to assemble micro motors; a large number of these micro motors are utilized as a part of economically accessible projection show frameworks, for example, the Texas Instruments Digital Micro mirror Device. Small scale engines, consolidated with Smart Dust, raise the between testing plausibility of making engineered creepy crawlies (see the "Micro robotics" sidebar) 


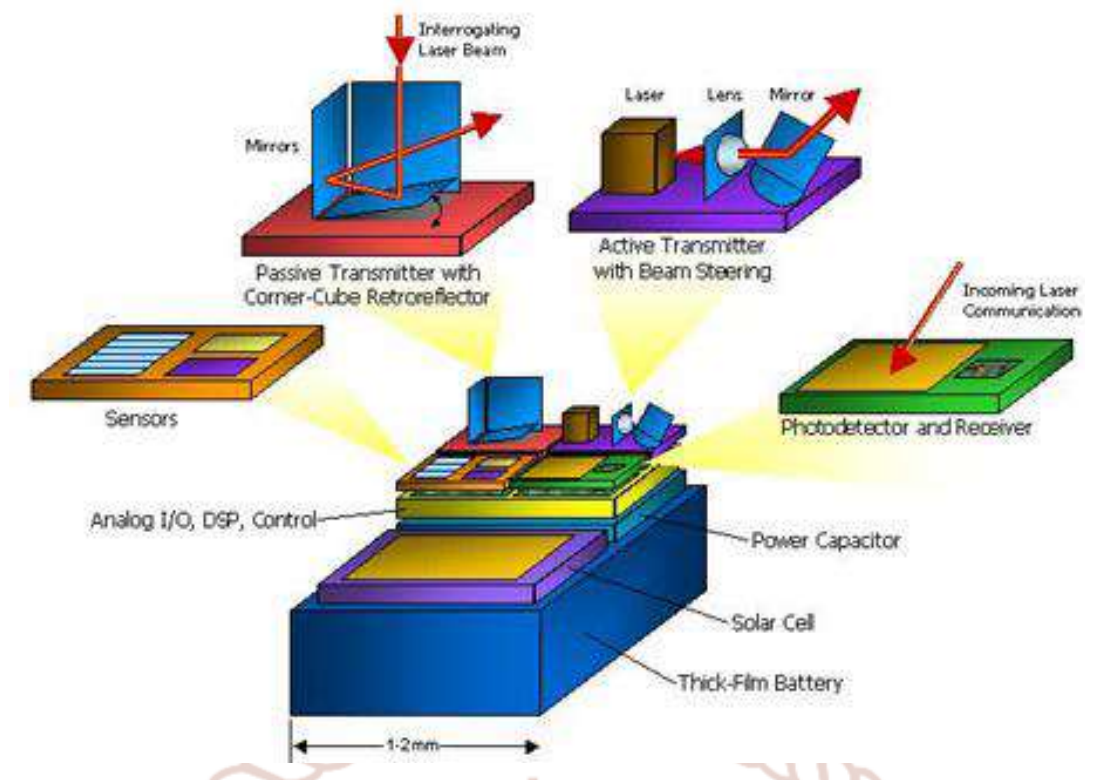

IV. COMPUTING AT THE MILLIMETER SCALE

Conventional PC engineering configuration has concentrated on diminishing a given task's execution time. To accomplish this objective, engineers have enhanced semiconductor-tor preparing exponentially, expanding the transistors "e speed while diminishing their size, in this way permitting more mind boggling designs that utilization expanded parallelism on a solitary pass on. Interestingly, figuring in an independent cubic-millimetre bundle must concentrate on limiting a given task's vitality utilization. Littler, speedier transistors have lessened parasitic capacitance, in this way bringing about reduced element control utilization. Steady electric-field scaling has lessened supply volt-ages, creating sensational power decreases for both superior and low-vitality registering in light of the fact that dynamic power has a quadratic reliance on supply voltage. Be that as it may, steady electric-field scaling additionally requires a diminishment in the limit voltage. This will bring about bigger spillage streams, which are as of now a worry in the elite processors to be discharged in 2001 that will spill amps of current. Accordingly, prepare engineers need to keep spillage mongrel leases low, which will likewise profit lowvitality architects. In millimetre-scale processing, the contracting transistor's estimate gives architects a chance to smaller huge figuring power into this little range. For instance, the Intel 8088 centre, initially created in a 3-micron process, would just require 0.12 -square millimetre in the wake of contracting lithographically into a current 0.18 -micron prepare, with a relating 100 lessening in vitality/guideline.

\section{T/ LOW-ENERGY COMPUTATION}

Other than cutting edge micro fabrication innovation forms, utilizing different procedures at each level accomplishes low-vitality calculation. In the first place, since we utilize a superior procedure yet work at low speeds, we can drop the supply voltage to the smaller than expected mum level at which the gadgets still capacity; theoretically this is 0.1 volt, 6 yet for 0.5 -to 0.2 -micron forms it is all the more sensibly 0.2 to 0.3 volt. To minimize current spillage, which can bring about critical power utilization at the low clock rates and obligation cycles that these low-vitality designs utilize, we can expand the channel-to-source junction's invert inclination, in this manner expanding the limit voltage. At first, including two additional supply voltages in this bundle may appear to be burdensome; in any case, if the bit searches sun based power, setting two little photodiodes on the incorporated circuit gives the few atoms per gadget important to predisposition these intersections. Different low-control format, circuit, and rationale level systems have been published. Figure 2 demonstrates an outcome of utilizing these techniques - the most pessimistic scenario vitality utilization of a 8 -bit viper in a 0.25 -micron prepare. The Smart Dust mote's assignments nearly identify with the physical domain, where the quickest inspecting is 10 to $20 \mathrm{kHz}$ for vibration and acoustic sensors so the measure of information is sufficiently little that we can utilize low information trans-mission rates. In this way, we can utilize check rates in the 1to $100-\mathrm{kHz}$ range to diminishing element control consumption. Regardless of these low clock rates, the circuits play out every one of their moves amid a little 
segment of the cycle; then they stay sit. Accordingly, shutting down squares for even a couple clock cycles spares vitality.

\section{REMOTE PROGRAMMABILITY}

A self-governing cubic-millimetre platform's computing necessities rely on upon the objective application on the grounds that committed equipment arrangements normally consume less vitality than a product arrangement. To avert unessential power utilization, we have to decide the base measure of programmability vital for a helpful stage. The essential bit occasionally tests at least one sensors, stores the qualities in memory, tunes in to an approaching parcel, and transmits current or put away information. Since transmitting the information and examining the sensors devour more vitality than playing out a computation, we can include more calculation, for example, thresholding, separating, phantom analysis, classification, Doppler move assurance, and encryption to enhance memory utilize and decide the noteworthiness of readings - along these lines giving more intelligent testing rates and decreasing the information transmission volume. Remote programmability assumes an essential part in millimetre-scale registering. Given their little size and huge numbers, we want to program these gadgets as once huge mob, without direct associations. Remote star gram ability likewise maintains a strategic distance from the expenses of recalling and reinventing gadgets after we convey them.

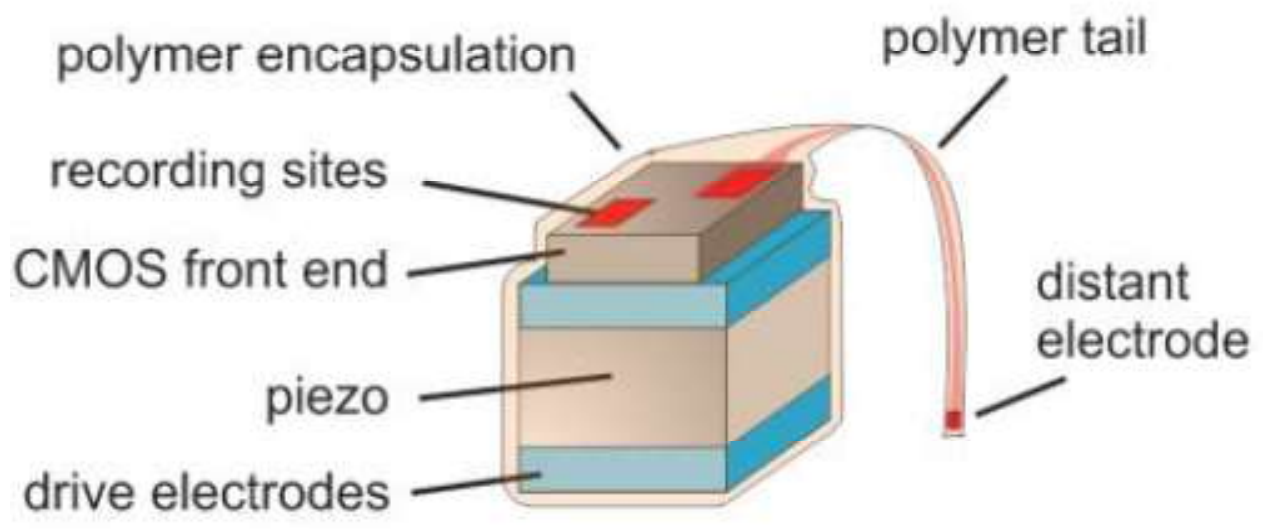

\section{Conveying FROM A GRAIN OF SAND}

Shrewd Dust's maximum capacity must be achieved when the sensor hubs speak with each other or with a focal base station. Remote correspondence encourages concurrent information gathering from a great many sensors. There are a few alternatives for imparting to and from a cubic-millimetre PC. Radio-recurrence and optical correspondences each have their qualities and shortcomings. Radio-recurrence correspondence is well under-stood, yet at present requires least power levels in the different mill watt run because of simple blenders, channels, and oscillators. In the event that bristle thin receiving wires of centimetre length can be acknowledged as a piece of a clean bit, then sensibly proficient reception apparatuses can be made for radio-recurrence correspondence. While the littlest finish radios are still on the request of a couple of hundred cubic millimetres, there is dynamic work in the scholarly world and industry to deliver cubicmillimetre radios. Semiconductor lasers and diode collectors are inherently little, and the comparing transmission and identification hardware for on/off keyed optical correspondence is more amiable to lowcontrol operation than most radio mapping. Maybe most vital, optical power can be collimated in tight pillars even from little openings. Diffraction authorizes a major farthest point on the dissimilarity of a bar, regardless of whether it originates from a radio wire or a focal point. Laser pointers are shoddy cases of mill radian collimation from a millimetre gap. To get comparable collimation for a $1-\mathrm{GHz}$ radio-recurrence flag would require a reception apparatus 100 meters over, because of the distinction in wavelength of the two transmissions. Therefore, optical transmitters of millimetre size can get recieving wire additions of one million or more, while correspondingly measured radio-recurrence reception apparatuses are destined by material science to be for the most part isotropic. Collimated optical correspondence has two noteworthy downsides. Observable pathway is required for everything except 
the most brief separations, and limited pillars infer the requirement for precise indicating. Of these, the guiding exactness can be unravelled by MEMS innovation and shrewd calculations; however an optical transmitter under a leaf or in a shirt pocket is of little use toward anybody. We have investigated optical correspondence in some profundity because of the potential for outrageous low-control correspondence.

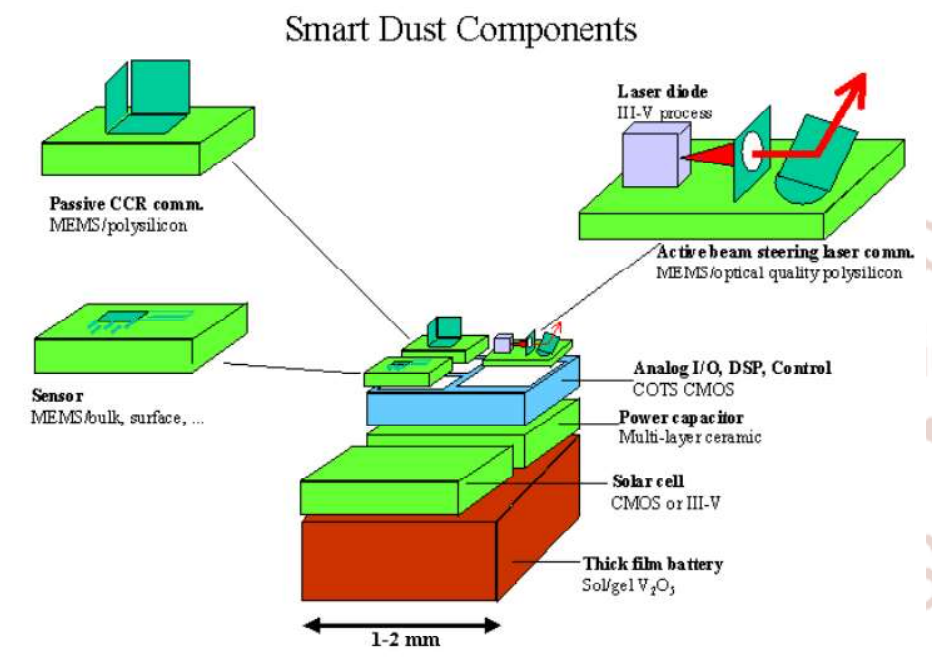

\section{OPTICAL COMMUNICATIONS}

We have investigated two ways to deal with optical interchanges: uninvolved intelligent frameworks and dynamic guided laser frameworks. In an aloof correspondence framework, the tidy bit does not require an installed light source. Rather, an extraordinary design of mirrors can either reflect or not reflect light to a remote source; this methodology looks like how a heliograph administrator skips daylight off a mirror to streak a Morse code message to ships - a thought followed to the fifth century BC, when the Greeks utilized reflected daylight as a reference point flag. Figure 3 demonstrates the cornershape retro reflector (CCR)10 used to adjust this thought to Smart Dust. Originators have utilized this gadget, however on a macro scale, for quite a long time in laser go discovering applications. A comparable gadget helped researchers decide the moon's separation from Earth.

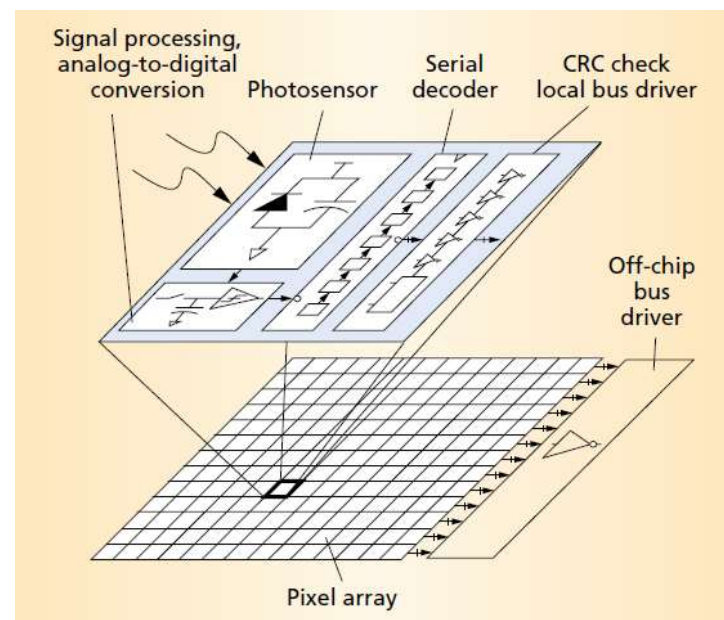

\section{Detached REFLECTIVE SYSTEMS}

In its least complex uninvolved design, the aloof intelligent gadget comprises of three commonly orthogonal mirrors. Light enters the CCR, skips off each of the three mirrors, and is reflected back parallel to the heading it entered. In the MEMS form, the gadget has one mirror mounted on a spring at a point marginally to one side from oppositeness to alternate mirrors. In this position, on the grounds that the light entering the CCR does not return along a similar passage way, minimal light comes back to the source-an advanced 0 . Applying voltage between this mirror and an anode underneath it makes the mirror to move to a position opposite different mirrors, subsequently bringing about the light entering the CCR to come back to its source - an advanced 1. The mirror's low mass permits the CCR to switch between these two states up to a thou-sand times each second, utilizing not exactly a nanojoule per $0 \rightarrow 1$ move. $\mathrm{A} 1 \rightarrow 0$ moves, then again, is basically free since dumping the charge put away on the terminal to the ground requires no vitality. Our most recent Smart Dust gadget is a $63-\mathrm{mm} 3$ selfsufficient bidirectional correspondence bit that gets an optical flag, produces a pseudorandom arrangement in light of this flag to imitate sensor information, and afterward optically transmits the outcome. The framework contains a small scale machined corner-3D square reflector, a $0.078-\mathrm{mm} 3$ correlative metal oxide semiconductor (CMOS) chip that draws 50 microwatts, and a listening device battery. Notwithstanding a battery-based operation, we have additionally controlled the gadget utilizing a2-mm2 sunlight based cell. This bit shows Smart Dust's basic ideas, for example, optical information trans-mission, information preparing, vitality administration, scaling down, and framework reconciliation. A latent correspondence framework endures a few restrictions. 
Not able to speak with each other, bits depend on a focal station furnished with a light source to send and get information from different bits. In the event that a given bit does not have an unmistakable observable pathway to the focal station, that bit will be secluded from the net-work. Additionally, on the grounds that the CCR reflects just a little part of the light radiated from the base station, this current framework's range can't without much of a stretch reach out past 1 kilometre. To bypass these restrictions, tidy bits must be dynamic and have their own installed light source.

\section{Dynamic STEERED LASER SYSTEMS}

For bit to-bit correspondence, a dynamic directed laser correspondence framework utilizes an installed light source to send a firmly collimated light bar toward a proposed beneficiary. Directed laser correspondence has the upside of high power thickness; for instance, a 1-milliwatt laser transmitting into 1 mill radian ( 3.4 bend seconds) has a thickness of around 318 kilowatts for every steradian (there are $4 \pi$ steradians in a circle), rather than a 100-watt light that emanates 8 watts for each steradian isotropic ally. A Smart Dust bit's radiate ted pillar would have a uniqueness of roughly 1 mill radian, allowing correspondence over colossal separations utilizing mill watts of force. Framing impromptu multihop systems is the most energizing utilization of bit to-bit correspondence. Multihop systems show critical difficulties to current system calculations-directing programming must upgrade every parcel's idleness as well as consider both the transmitter's and collector's vitality holds. Every bit should precisely measure the requirements to detect, register, convey, and assess its vitality hold status before apportioning valuable nanojoules of vitality to turn on its transmitter or collector. Since these bits invest the majority of their energy dozing, with their collectors killed, planning a typical conscious time over the system is troublesome. In the event that bits don't wake up in a synchronized way, a very dynamic system topology and substantial parcel inertness result. Utilizing burst-mode correspondence, in which the laser works at up to a few several megabits for each second for a couple of milliseconds, gives the most vitality proficient approach to timetable this system. This methodology limits the bit's obligation cycle and better uses its vitality holds. The directed light-footed laser transmitter comprises of a semi-conductor diode laser combined with a collimating focal point and MEMS shaft controlling optics in view of a two-level ofopportunity silicon micro mirror, as Figures 4 and 5 appear. This framework coordinates every single optical segment into a dynamic 8-mm3 volume.

\section{Tuning in TO A DUST FIELD}

Many Smart Dust applications depend on direct optical correspondence from a whole field of clean bits to at least one base stations. These base stations should consequently have the capacity to get a vast volume of concurrent optical transmissions. Encourage, correspondence must be conceivable outside in splendid daylight which has a force of around 1 kilowatt for every square meter, despite the fact that the clean bits each transmit data with a couple of mill watts of force. Utilizing a thin band optical channel to wipe out all daylight with the exception of the segment close to the light recurrence utilized for correspondence can mostly tackle this second issue, yet the surrounding optical power frequently stays substantially more grounded than the got flag control.

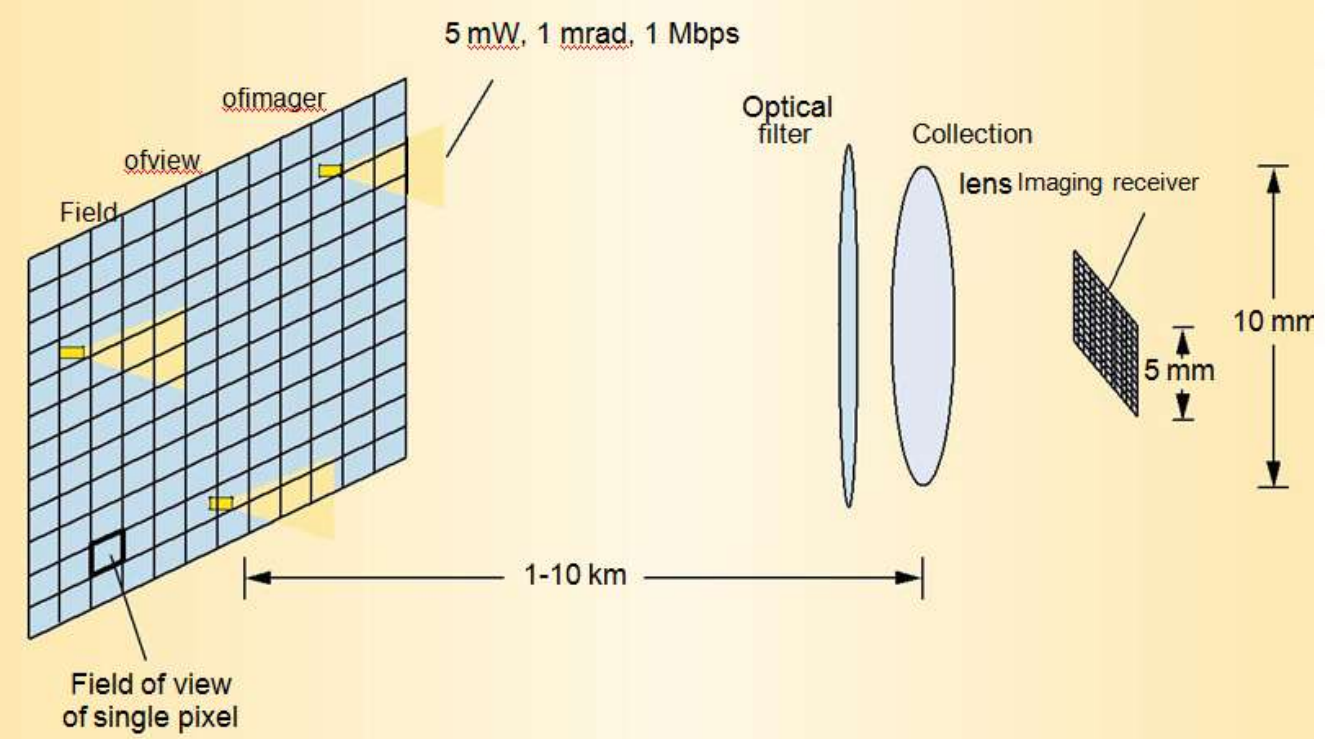




\section{Favourable circumstances OF IMAGING RECEIVERS}

Similarly as with the transmitter, the short wavelength of optical transmissions contrasted and radio frequencies defeats both difficulties. Light from a substantial field of view can be engaged into a picture, as in our eyes or in a camera. Imaging beneficiaries use this to investigate diverse segments of the picture independently to prepare concurrent transmissions from various edges. This technique for recognizing transmissions in view of their beginning area is alluded to as space division different get to (SDMA). Conversely, most radio-recurrence receiving wires get all occurrence radio power in a solitary flag, which requires utilizing extra strategies, for example, recurrence tuning or code division numerous get to (CDMA), to isolate synchronous transmissions. Imaging recipients additionally offer the benefit of drastically diminishing the proportion of surrounding optical energy to got flag power.11 ideally; the imaging collector will concentrate the majority of the got control from a solitary transmission onto a solitary photograph identifier. In the event that the beneficiary has a $\mathrm{n} \square \mathrm{n}$ exhibit of pixels, then the surrounding light that every pixel gets is lessened by a variable $\mathrm{n} 2$ contrasted and a no imaging collector. Normally, utilizing an incentive for $\mathrm{n}$ in the vicinity of 8 and 32 makes the surrounding light power immaterial contrasted and the electronic commotion in the simple hardware.

\section{Camcorder}

A camcorder is a clear execution of an imaging collector. In the event that every part in a province of Smart Dust bits flashes its own flag at a rate of a couple of bits for every second, then every transmitter will show up in the video stream at an alternate area in the picture. We have actualized such a framework utilizing a tablet with an edge grabber that procedures an ongoing video motion in programming. We tried this framework to transmit climate data from Twin Peaks in San Francisco to a camcorder in Berkeley, 21.4 kilometres crosswise over San Francisco Bay. The transmitter comprises of a one-cubic-inch Smart Dust bit taunt up that adjusts a conventional red laser pointer at a couple of bits for every second, utilizing just 3.5 mill watts of pinnacle optical transmission control in a 2-milliradian cone.12 Smart Dust's enhanced framework will permit comparative connection separations, with the additional preferred standpoint of computerized transmitter-recipient arrangement Utilizing a rapid camera and a devoted computerized flag processor to prepare the video flag accomplishes higher information rates. With current cameras and DSPs, master cessing video at around 1,000 casings for each second ought to be plausible. This would permit correspondence at a couple

\section{REFERENCES}

1. J. Kahn, R.H. Katz, and K. Pister, "Emerging Challenges: Mobile Networking for Smart Dust," J. Comm. Net-works, Sept. 2000, pp. 188-196.

2. C. Piguet et al., "Low-Power Design of 8-b Embedded CoolRisc Microcontroller Cores," IEEE J. Solid-State Circuits, July 1997, pp. 10671078

3. http://www.ubiq.com/hypertext/weiser/ acmfuture2endnote.htm, 1996. 